\title{
ANÁLISE ERGONÔMICA NO PROCESSO DE EXTINÇÃO DA Ca(OH)
}

\section{ERGONOMIC ANALYSIS IN THE EXTINCTION CA $(\mathrm{OH})_{2}$ PROCESS}

\author{
Camila Gávio Coutinho ${ }^{1}$; Francisco Santos Sabbadini ${ }^{2}$; Mitaiana Maria de Carvalho Pinto ${ }^{3}$; Nelson \\ Tavares Matias ${ }^{4}$; Rosinei Batista Ribeiro ${ }^{5}$ \\ ${ }^{1}$ Universidade do Estado do Rio de Janeiro - UERJ - Resende - Brasil \\ mila_gavio@hotmail.com.br \\ ${ }^{2}$ Universidade do Estado do Rio de Janeiro - UERJ - Resende - Brasil \\ franciscosabbadini@gmail.com \\ ${ }^{3}$ Universidade do Estado do Rio de Janeiro - UERJ - Resende - Brasil \\ mitaiana@hotmail.com.br \\ ${ }^{4}$ Universidade do Estado do Rio de Janeiro - UERJ - Resende - Brasil \\ nelson.matiaz@gmail.com \\ ${ }^{5}$ Faculdade de Tecnologia do Estado de São Paulo - IES - Cruzeiro - Brasil \\ rosinei1971@gmail.com
}

\begin{abstract}
RESUMO
Reduzir gastos, associado a um ambiente de trabalho auspicioso é o foco para um produto de mais qualidade e bem-estar, proporcionando assim competitividade e atratividade aos consumidores finais. Na indústria química, a redução dos impactos ergonômicos e ambientais durante um processo é de grande importância por minimizar danos à empresa, trabalhadores, comunidade e meio ambiente, levando a uma qualidade de vida em toda a cadeia. Sendo assim o objetivo do projeto foi avaliar o processo de extinção de $\mathrm{Ca}(\mathrm{OH}) 2$ para tratamento de água bruta. Dentre os principais problemas, temos a alta exposição do colaborador com o particulado que emana no ar, o excesso de movimentação para realização da atividade e perda de água/produto para a limpeza da área após o processo. O processo inteiro foi tomado como um estudo de caso e para o seu desenvolvimento aplicou-se a Análise Ergonômica do Trabalho concomitantemente as ferramentas RULA, REBA, NIOSH e NIOSH 0600.
\end{abstract}

Palavras-chave: $\mathrm{Ca}(\mathrm{OH})_{2}$; Análise Ergonômica; Análise Ambiental.

\section{Introdução}

Com a evolução tecnológica e a crescente concorrência nos mais diversos setores industriais, tanto no Brasil como no mundo, as empresas têm buscado valorizar sua mão de obra oferecendo, entre outras coisas, melhores condições de trabalho. A preocupação com a saúde do trabalhador precisa ser entendida como uma ferramenta para melhorar a qualidade de vida do mesmo no seu posto de trabalho e não como uma exigência legal apenas, assim como a preocupação com o meio ambiente 
pode prevenir e alertar condições ambientais que poderão ser nexos causais em litígios trabalhistas (EHS, 2014).

Nesse contexto, avaliando um ambiente ou posto de trabalho, enquadra-se a ergonomia que, segundo a Associação Brasileira de Ergonomia (ABERGO, 2000), é uma disciplina científica da interação do homem com o sistema e a aplicação de ferramentas para otimização do bem estar e desempenho global do sistema a fim de adaptar o trabalho ao homem e não o inverso.

Tão importante quanto os aspectos ergonômicos, são os aspectos ambientais, pois ambos podem representar prejuízos econômicos para a empresa, seja pela perda de produtividade e surgimento de doenças ocupacionais quanto pelo desperdício de matérias primas, água, energia, gasto com tratamento, armazenamento e disposição final dos resíduos.

Atualmente, interpretar as Estações de Tratamento de Efluentes e as Estações de Tratamento

de Água como setores industriais que prestam serviços por meio de um sistema produtivo e de transformação, mostra que estas podem enfrentar problemas de caráter ergonômico, de Higiene e Segurança do Trabalho, como qualquer outro setor dentro de uma empresa. O Código Nacional de Atividade Econômica (CNAE) que engloba trabalhadores de coleta e tratamento de água acaba por não revelar um número exato de trabalhadores do setor, bem como os acidentes sofridos por esses, mostrando a desconfiança por parte das empresas em apresentar dados relacionados à segurança (BUDA, 2004, p. 145).

\section{Objetivos do estudo}

Melhorar as condições do colaborador, mitigando ou até eliminando os possíveis danos ao mesmo, durante o processo da extinção da Cal Hidratada. Este consiste desde a chegada da matéria prima através de sacarias que são fracionadas para caberem no elevador de carga, passando para diluição da mesma em tanques apropriados, até a lavagem da área no término do processo.

Consequentemente tem-se melhoria da qualidade do processo, e contribui para a empresa reduzir o consumo de água e geração de efluentes a fim de atingir suas metas globais.

\section{Metodologia adotada}

O presente trabalho utilizou como método a abordagem da Análise Ergonômica do Trabalho, apoiada por ferramentas tais como: National Institute for Occupational Safety and Health (NIOSH), Rapid Entire Body Assessement (REBA), Rapid Upper Limb Assessment (RULA), American Conference of Industrial Hygienists (ACGIH) são pertinentes. Também foram realizadas pesquisas nas diversas literaturas como livros, revistas científicas, artigos, teses, dissertações e material disponível na internet. 
Aplicativos tais como o Kinovea e Softwares como o Ergolândia, foram empregados no decorrer do processo, sendo fundamentais para análises quantitativa e qualitativa muito mais precisas.

A Figura 1 demostra o progresso de todo o estudo e trabalho desenvolvido em questão.

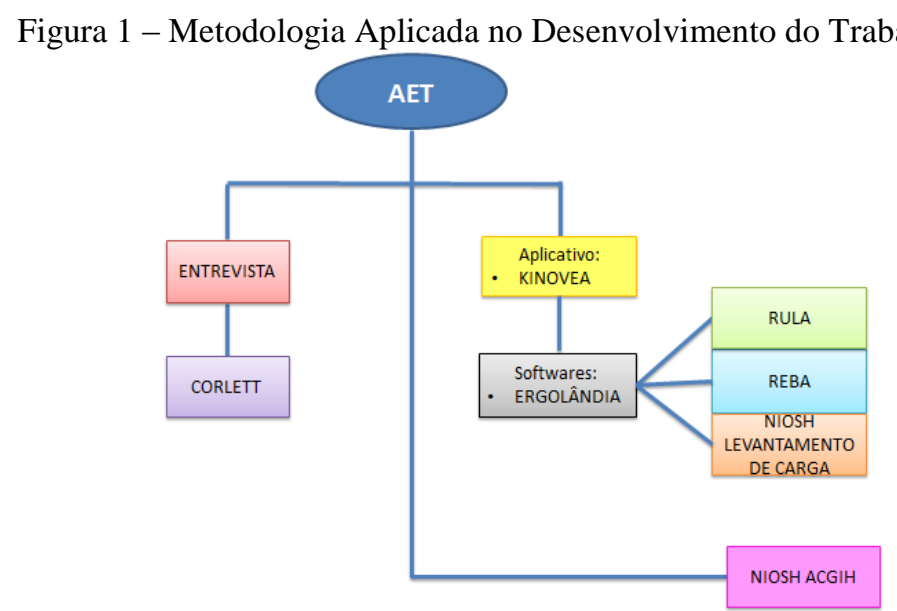

Fonte: COUTINHO e PINTO, 2014

\section{Estudos de pesquisa}

Através de estudos realizados no processo químico utilizado para a extinção da Cal hidratada $\mathrm{Ca}(\mathrm{OH})_{2}$, assim como nas ferramentas ergonômicas atualmente disponíveis para análise foi possível entender os pontos críticos e levantar possíveis soluções de melhoria.

Extinção de $\mathbf{C a}(\mathrm{OH})_{2}$ : A extinção de $\mathrm{Ca}(\mathrm{OH})_{2}$ é necessária para o tratamento da água bruta em Estações de Tratamento de Água. Todavia sua importância não deve ser superestimada em relação à saúde dos colaboradores envolvidos em todo o processo, já que este acaba por envolver movimentação das sacarias exigindo mudanças intermitentes de postura e levantamento de peso, além da inalação de particulados durante o processo de extinção.

\footnotetext{
Os pulmões e o cérebro são muito atacados nesses obreiros, sobretudo os pulmões que aspiram, junto com o ar, exalações minerais, resultando daí os primeiros agravos, pois aquelas emanações se introduzem no órgão vital e se misturam com o sangue, alterando e arruinando a constituição natural do cérebro e do fluído nervoso [...]. (RAMAZZINI, 1700 apud FUNDACENTRO, 2001).
}

\section{AET - Análise Ergonômica do Trabalho}

A Análise Ergonômica de Trabalho é um método que se subdivide em análise da demanda, tarefa e atividade para fazer o diagnóstico e as recomendações necessárias (ORMELEZ; ULBRICHT, 2010, p. 71). Este método se refere à NR-17 de forma abrangente, com um estudo detalhado dos postos de trabalho para que depois de detectados os fatores de riscos ocupacionais, as soluções ergonômicas sejam implementadas na empresa. (VIEIRA, 2014) 
“A abrangência pode ser classificada em: análise de sistemas, análise do posto de trabalho e análise do trabalho propriamente dito" (SILVA et al, 2011).

“Analisar a atividade significa reconstruir a lógica dos trabalhadores em seu próprio curso da ação a partir de observações objetivas, que permitam aprender o subjetivo e explicitar as razões de um determinado comportamento", para Abrahão et al (2009, p. 37).

A análise de sistemas, conjunto de elementos que interagem entre si, deve ser realizada a partir de uma visão ampla, considerando todos os elementos que irão interferir direta ou indiretamente no desempenho da máquina dentro de seu ambiente de operação. A qualidade desse desempenho influenciará o grau de adaptação do operador, do sistema produtivo, da organização de produção e até a cultura do país e região, de acordo com lida (2003, p. 16).

Segundo Falzon (2007), a análise ergonômica no posto de trabalho precisou evoluir para se tornar muito mais ampla em um ambiente técnico e social, colocando novas questões de âmbito de situações de trabalho e sua concepção dentro da dimensão coletiva, das interações sociais e das interações entre diferentes equipamentos de trabalho.

Para Costa (2014), as bases teóricas da análise ergonômica de postos vêm de entre outras da biomecânica ocupacional, da psicologia da informação, da higiene industrial e de um modelo sócio técnico de organização do trabalho, podendo resultar de recomendações gerais e de objetivos para a segurança e saúde no trabalho.

A análise do trabalho é o estudo das situações, dos operadores, da obtenção de informações sobre os deslocamentos, posturas e esforços, métodos de trabalho e movimentos repetitivos que podem condicionar o nível de produtividade e de qualidade (SILVA et al, 2011). Não se deve confundir o trabalho prescrito que é a tarefa, ou seja, o que é prescrito pela empresa exterior ao trabalhador com o trabalho real, que é a atividade, podendo ser vista como o que o trabalhador faz para atingir os objetivos definidos na tarefa (ABRAHÃO et al, 2009, 48-52).

Os estudiosos que praticam a Ergonomia devem ter amplo conhecimento da disciplina, levando em consideração os fatores físicos, cognitivos, sociais, organizacionais, ambientacionais e outros ainda (FALZON, 2007, p. 5). (Quadro 1)

Quadro 1 - Tipos da Ergonomia

\begin{tabular}{|l|l|}
\hline Ergonomia & $\begin{array}{l}\text { A ergonomia física trata das características anatômicas, antropométricas, fisiológicas e } \\
\text { biomecânicas do homem em sua relação com a atividade física. Os temas mais relevantes } \\
\text { comprica }\end{array}$ \\
\hline $\begin{array}{l}\text { Erobondem as posturas de trabalho, a manipulação de objetos, os movimentos repetitivos, os } \\
\text { Cognitiva }\end{array}$ & $\begin{array}{l}\text { A ergonomia cognitiva trata dos processos mentais, tais como a percepção, a memória o } \\
\text { raciocínio e as respostas motoras, com relação às interações entre as pessoas e outros } \\
\text { componentes de um sistema. Os temas centrais compreendem a carga mental, os processos de }\end{array}$ \\
\hline
\end{tabular}




\begin{tabular}{|l|l|l|} 
& $\begin{array}{l}\text { decisão, o desempenho especializado, a interação homem-máquina, a confiabilidade humana, } \\
\text { o estresse profissional e a formação, na sua relação com a concepção pessoa -sistema. }\end{array}$ \\
\hline Ergonomia & $\begin{array}{l}\text { A ergonomia organizacional trata da otimização dos sistemas sociotécnicos, incluindo sua } \\
\text { estrutura organizacional, regras e processos. Os temas mais relevantes compreendem a } \\
\text { Organizacional } \\
\text { comunicação, a gestão dos coletivos, a concepção do trabalho, a concepção do trabalho, a } \\
\text { ergonomia comunitária, o trabalho cooperativo, as novas formas de trabalho, a cultura } \\
\text { organizacional, as organizações virtuais, o teletrabalho e a gestão pela qualidade. }\end{array}$ \\
\hline Ergonomia & $\begin{array}{l}\text { Trata-se dos ambientes sonoros, visuais, térmicos, de pressão (hipo e hiperbáricos, sem } \\
\text { gravidade). A fisiologia traz a essa área uma contribuição essencial, que leva às vezes à } \\
\text { definição de normas de conforto. Ela contribui para a concepção de locais de trabalho } \\
\text { adaptados e eventualmente de meios de proteção eficazes. }\end{array}$ \\
\hline
\end{tabular}

Fonte: Adaptado de FALZON (2007, p. 05-35)

Ferramentas ergonômicas: Segundo Matias (2011, p. 26) existem métodos ergonômicos capazes de avaliar, medir e quantificar qualitativamente e quantitativamente uma atividade. Para o presente trabalho as ferramentas Rapid Upper Limb Assessment (RULA), Rapid Entire Body Assessment (REBA) e National Institute for Occupational Safety and Health (NIOSH) foram analisadas e utilizadas. Essas ferramentas seguem listadas abaixo.

\section{a) Determinação rápida dos membros superiores (RULA)}

O método de Determinação Rápida dos Membros Superiores (Rapid Upper Limb Assessment - RULA) foi desenvolvido por Dr. Lynn McAtamney e Dr. Nigel Corlett. (LUEDER, 1996).

Segundo McAtamney e Corlett (1993, apud Stanton et al., 2004, p. 7-1) o método RULA fornece um fácil cálculo de classificação das cargas osteomusculares em tarefas em que o pescoço e os membros superiores correm risco. A ferramenta provê uma única nota como um snapshot da tarefa que classifica a postura, a força e os movimentos requeridos. O risco é calculado em pontuações entre 1 (baixo) e 7 (alto). Esses pontos são agrupados em 4 níveis de ação que indicam um quadro em que é razoável esperar que um controle dos riscos seja iniciado. Para Lueder (1996) uma baixa pontuação no método RULA não garante que o local de trabalho está livre de perigos ergonômicos, e uma alta pontuação não assume que graves problemas existem. Este método é desenvolvido para detectar posturas ou fatores de risco que merecem mais atenção.

Como informação de entrada, o método requer diagramas de posturas com pesos aditivos, retornando como resultado a classificação do posto de trabalho quanto à prioridade de intervenção (GUIMARÃES; NAVEIRO, 2004, p. 67). 
Como cita McAtamney e Corlett (1993 apud Stanton et al., 2004, p.7-1) as quatro principais atuações do método RULA são:

- Medição de risco musculoesquelético, usualmente como parte de uma ampla investigação ergonômica;

- Comparação do esforço musculoesquelético entre design da estação de trabalho atual e modificada;

- Avaliar resultados como produtividade ou compatibilidade de equipamentos;

- Orientar trabalhadores sobre riscos musculoesqueléticos criados por diferentes posturas de trabalho.

E ainda segundo os autores, o método é composto pelas 3 etapas descritas:

- Seleção da postura ou posturas para avaliação. Para Pavani e Quelhas (2006) apud Guimarães et al. (2012) nessa fase ocorre a divisão de dois grupos. O grupo A compreende os braços, antebraços e punhos. E o grupo B trata do pescoço, tronco e pernas. É analisado o ângulo de extensão de cada uma dessas partes do corpo humano em relação ao mobiliário do setor/ posto de trabalho e extinção.

- As posturas são pontuadas usando uma planilha de pontos, diagramas departes do corpo e tabelas;

- De acordo com uma escala de níveis de ação, as pontuações dos grupos A e B são acrescentadas notas relativas ao tipo de trabalho muscular, a repetitividade e ao nível de esforço, formando a pontuação final, conforme a Figura 8 (PAVANI;QUELHAS, 2006 apud GUIMARÃES et al 2012) Essas pontuações são convertidas em uma das quatro medidas propostas pela Tabela 1.

Tabela 1 - Níveis de intervenção para os resultados do método RULA

\begin{tabular}{lcll}
\hline Nível de Ação & Pontuação & Nível de Risco & Intervenção \\
\hline 1 & 1 a 2 & Negligenciável & $\begin{array}{l}\text { Postura aceitável, desde que } \\
\text { não seja mantida por longos } \\
\text { períodos }\end{array}$ \\
2 & 3 a 4 & Baixo & $\begin{array}{l}\text { Pode ser necessária }- \\
\text { investigar melhor }\end{array}$ \\
3 & 5 a 6 & Alto & Necessária em breve - \\
urgente investigar melhor & Necessária agora - \\
modificações urgentes
\end{tabular}

Fonte: Adaptado de PAVANI e QUELHAS (2006, apud DOMINGUES et al, 2012) 


\section{b) Avaliação corporal rápida (REBA)}

Já o método Rapid Entire Body Assessment (REBA), foi criado para julgar posturas de trabalhos não previsíveis. Desenvolvido por Sue Hignett e Lynn McAtmney, foi publicado na Applied Ergonomics em 2000 (BAÚ, 2002; DIEGO-MÁS; CUESTA, 2007; HIGNETT; MCATSMNEY, 2005).

De acordo com seus criadores Hignett e McAtmney (2005), a ferramenta possibilita a análise das posturas utilizadas no dia a dia do trabalho, de esforços aplicados, de tipos de movimentos ou ações, atividade muscular, repetição do trabalho e o tipo de pegada adotada pelo trabalhador ao realizar a tarefa.

Segundo os estudiosos Diego-Más e Cuesta (2007), o REBA avalia tanto posturas em movimentos e estáticas, como também posições inesperadas e mudanças rápidas de postura. Segundo Hignett e McAtamney (2000, apud BATIZ; VERGARA; LICEA, 2012), o método REBA apresenta uma similaridade ao método RULA, estando basicamente direcionadas na análise de atividades com movimentos repetitivos e em relação aos membros superiores e inferiores.

O método REBA consiste na divisão das regiões do corpo em dois grupos, os quais são: 'A', em que se analisa o pescoço, o tronco e as pernas; e 'B', em que são analisados os braços, antebraços e punhos. Leva-se em consideração que cada etapa do método é constituída de uma única postura. A análise de cada posto de trabalho se dá a partir da postura mais habitualmente utilizada pelo avaliado. Assim, a reunião dos resultados caberá ao avaliador chegar a uma conclusão do posto, indicando o nível de risco do posto mostrando se pode haver ou não o aparecimento de lesões.

A pontuação é feita em dois grupos, após isso, é feita a soma dos pontos de ambos os grupos, dando origem à pontuação final da postura. É comparado então, o resultado obtido ao nível de ação necessária. O nível de ação é dividido em 5 (cinco) etapas, em que o menor é o 0 (zero), correspondente ao intervalo de movimento ou postura de trabalho aceitável e que não necessita de melhorias na atividade; e o último nível é o 4 (quatro), em que o fator de risco é considerado muito alto, sendo necessária a atuação imediata. Esses níveis são revertidos na Tabela 2 abaixo: Inscrição em faculdades locais, 2005

Tabela 2 - Níveis de intervenção para os resultados do método REBA

\begin{tabular}{lcll}
\hline Nível de Ação & Pontuação & Nível de Risco & Intervenção \\
\hline 0 & 1 & Negligenciável & Desnecessária \\
1 & 2 a 3 & Baixo & Pode ser necessária \\
2 & 4 a 7 & Médio & Necessária \\
3 & 8 a 10 & Alto & Necessária em breve
\end{tabular}




\begin{tabular}{llll}
\hline 4 & 11 a 15 & Muito Alto & Necessária agora
\end{tabular}

Fonte: Adaptado de PAVANI e QUELHAS (2006, apud DOMINGUES et al, 2012)

O REBA definirá se o avaliador tem necessidade de replanejar o posto para diminuir ou acabar com determinadas posturas que prejudiquem o trabalhador. Alinhado a essa avaliação, as pontuações obtidas em cada segmento do corpo, o esforço com cargas e as atividades em geral, guiará o avaliador nos aspectos que apresentam maiores problemas ergonômicos com a finalidade de intervir por meio de ações preventivas. (DIEGO-MÁS; CUESTA, 2007).

Outro ponto que se deve destacar sobre o método é que, por ser codificado individualmente por meio da divisão de segmentos, permite a avaliação de cada segmento (risco de lesões, atividade muscular dinâmica ou estática, posturas instáveis e mudanças rápidas de postura), identificando a necessidade de uma intervenção mais rápida e eficiente. Vale ressaltar que o método não considera tópicos como vibração e gasto alto de energia por parte do trabalhador.

\section{c) NIOSH - Instituto Nacional de Segurança e Saúde Ocupacional (National Institute for Occupational Safety and Health)}

National Institute for Occupational Safety and Health ou NIOSH (Instituto Nacional de Segurança e Saúde Ocupacional) é responsável pela realização de pesquisas e produção de recomendações para a prevenção de lesões e doenças relacionada com o trabalho (CDC, 2014).

Foi levado em consideração no estudo de caso o NIOSH para levantamento manual de cargas e NIOSH para partículas respiratórias.

- NIOSH para levantamento manual de cargas:

Para quantificar o levantamento de peso, a ferramenta National Institute for Ocupational Safety and Health (NIOSH) foi utilizada. Em alguns postos de trabalho muitas das vezes os profissionais da área de saúde ocupacional se veem com a necessidade de tornar exprimível em quantidade algumas situações. Dentre elas, para Agahnejad (2011), a que possui muito destaque é a qualificação do levantamento manual de cargas, pois, mesmo nos tempos modernos de hoje, esta é ainda uma das maiores causas de problemas musculoesqueléticos nos trabalhadores.

Por conta dessas necessidades, em 1980 o NIOSH, dos Estados Unidos, fez um patrocínio para o desenvolvimento de um critério de avaliação para o levantamento manual de cargas, o qual foi revisado em 1991. Segundo Okimoto (2002), dentre o que foi proposto pelo grupo relacionado ao estudo, dois indicadores foram estipulados: Limite de Peso Recomendado (LPR) e Índice de Levantamento (IL), sendo utilizados como parâmetros para avaliação das chances de ocorrer uma lesão de coluna no trabalhador quando estiver realizando uma operação de movimentação de cargas. 
Esses indicadores foram utilizados na obtenção de uma equação desenvolvida, tendo com base os critérios biomecânicos, fisiológicos e psicofísicos.

- NIOSH para partículas respiratórias:

Atualmente, uma das preocupações mais relevantes dos técnicos de higiene e segurança do trabalho é a exposição dos trabalhadores a agentes químicos no ar do ambiente profissional. Normalmente focaliza-se a análise em agentes químicos sólidos, vulgarmente chamados de poeiras ocupacionais, oriundos de processos produtivos em diversos tamanhos.

Uma das metodologias utilizadas na Avaliação da Exposição Profissional e Poeiras Totais é a que consta na referencial NIOSH 0500 e 0600, tendo por fundamento a gravimetria esta apresenta orientações para a determinação da concentração a que os trabalhadores estão expostos (NIOSH, 1994). O Instituto Português de Acreditação no âmbito as amostragem de poeiras totais no ar ambiente laboral propõe o mesmo referencial (IPAC, 2009).

Para fazer a medição das partículas suspensas no ar, precisa-se, anteriormente, completar três estágios prévios, os quais são:

Lista de agentes químicos: Será solicitada uma lista de todos os agentes químicos envolvidos no ambiente profissional em questão, como matéria-prima, produtos intermediários, finais, de reações, entre outros.

Identificação dos fatores inerentes ao local de trabalho: Os processos e procedimentos de trabalho serão analisados a fim de determinar o potencial para a exposição a agentes químicos, como processo de produção, fontes de emissão, tempo de exposição, entre outros.

Apreciação das exposições: Este estágio propõe a integração da identificação das exposições potenciais e os fatores do local.

Após o último estágio, é feita a medição, onde será analisado se a exposição do trabalhador aos agentes químicos no ambiente profissional está perto dos valores limites aceitáveis. Como meio de avaliação, uma proposta feita pelo National Institute for Ocupational Safety and Health (NIOSH) pode ser observada na Tabela 3 abaixo:

Tabela 3 - Valores limites de exposição

\begin{tabular}{lcc}
\hline Parâmetros Avaliados & $\begin{array}{l}\text { VLE-MP } \\
\left(\mathbf{m g} / \mathbf{m}^{\mathbf{3}}\right)\end{array}$ & $\begin{array}{c}\text { Metodologia de } \\
\text { amostragem (*) }\end{array}$ \\
\hline Partículas sem outra classificação (PSOC) & & \\
Partículas inaláveis (totais) & 10 & NIOSH 0500 (4) \\
Partículas respiráveis & 3 & NIOSH 0600 (3)
\end{tabular}

(*) Nestes métodos encontram-se definidos os caudais de ar a amaostrar, volumes máximo e mínimo, características dos filtros a utilizar, entre outras características.

Fonte: BARBOSA; MATOS; SANTOS, 2014 


\section{Resultados e discussão}

Após o estudo das ferramentas ergonômicas e compreensão do processo foram coletados dados através de entrevistas informais, questionários, fotografias e vídeos. Todos os dados passaram por análise, no caso das entrevistas os resultados foram quantificados e no caso das fotos e os vídeos foram analisados através de softwares, como Kinovea e Ergolândia.

O Kinovea é um aplicativo portador de recursos que permitem avaliar os detalhes registrados durante uma AET. O aplicativo permite aproximar áreas específicas da imagem, ressaltando todos os pontos importantes, além de disponibilizar marcadores: ângulos, cronômetro, grade, perspectiva, legenda, marcador em cruz entre outros.

Após a utilização do Kinovea, o software Ergolândia pôde ser aplicado. Este possui 20 ferramentas ergonômicas para avaliação e melhoria dos postos de trabalho, aumentando sua produtividade e diminuindo os riscos ocupacionais, foi usado no trabalho. Dentre as ferramentas que o software dispõe as metodologias RULA, REBA e NIOSH para levantamento de peso foram aplicadas.

A partir de então, com os dados obtidos consistidos pôde-se atentar para a obtenção do diagnóstico.

\section{Resultado do Questionário}

Após uma entrevista informal, foi apresentado um questionário, em que se obteve como resposta os dados apresentados no Quadro 2.

Quadro 2- Relação Percentual Obtida Através do Questionário

\begin{tabular}{|c|c|c|c|c|c|c|}
\hline & $(1)$ & $(2)$ & (3) & $(4)$ & $(5)$ & $(6)$ \\
\hline Temperatura no posto de trabalho & - & - & - & $67 \%$ & $33 \%$ & - \\
\hline Ruído no posto de trabalho & - & - & $100 \%$ & - & - & - \\
\hline Iluminação no posto de trabalho & - & - & $67 \%$ & $33 \%$ & - & - \\
\hline Vibrações no posto de trabalho & - & - & $33 \%$ & $33 \%$ & $33 \%$ & - \\
\hline Poeiras no posto de trabalho & $33 \%$ & $33 \%$ & - & $33 \%$ & - & - \\
\hline Postura de trabalho adotada & - & - & $33 \%$ & $33 \%$ & $33 \%$ & - \\
\hline Qualidade de ferramentas e equipamentos de trabalho & - & - & $67 \%$ & $33 \%$ & - & - \\
\hline Tempo de pausas (inclusive intervalo para almoço e lanche) & - & - & $33 \%$ & $67 \%$ & - & - \\
\hline Folgas (descanso semanal) & - & - & - & $100 \%$ & - & - \\
\hline Relacionamento com colegas do setor & - & - & $67 \%$ & $33 \%$ & - & - \\
\hline Relacionamento com a chefia & - & - & $33 \%$ & $33 \%$ & - & $33 \%$ \\
\hline Oportunidade de uso das habilidades & - & - & - & $67 \%$ & $33 \%$ & - \\
\hline Atendimento a sugestões e solicitações & - & $33 \%$ & $33 \%$ & $33 \%$ & - & - \\
\hline Ritmo de trabalho & - & - & $33 \%$ & $67 \%$ & - & - \\
\hline Organização e distribuição das tarefas por parte da chefia & - & $33 \%$ & $33 \%$ & $33 \%$ & - & - \\
\hline Treinamento da função a ser realizada no posto de trabalho & - & - & $67 \%$ & $33 \%$ & - & - \\
\hline Os Equipamentos de Proteção Individual (EPI) disponibilizados são: & - & - & - & $33 \%$ & $67 \%$ & - \\
\hline O treinamento quanto ao uso dos EPI é: & - & - & - & $100 \%$ & - & - \\
\hline Esforço físico no posto de trabalho & - & - & $67 \%$ & $33 \%$ & - & - \\
\hline Esforço mental no posto de trabalho & - & - & $67 \%$ & $33 \%$ & - & - \\
\hline Monotonia no posto de trabalho & - & - & $33 \%$ & $67 \%$ & - & - \\
\hline
\end{tabular}


Criatividade no posto de trabalho

Dinamismo no posto de trabalho

Repetitividade no posto de trabalho

Diversificação de atividades no posto de trabalho

Envolvimento de responsabilidades no posto de trabalho

Valorização pessoal no posto de trabalho

Autonomia para realizar as atividades no posto de trabalho

A(s) instrução (ões) de serviço (IS) são clara e objetivas

Pressão psicológica por parte dos supervisores relacionada ao posto de trabalho

(1) Extremamente insatisfatório, (2) Insatisfatório, (3) Regular, (4) Satisfatório, (5) Extremamente satisfatório, (6) Nulo

Fonte: COUTINHO e PINTO, 2014

Verificou-se no diagrama de Corlett que $100 \%$ dos entrevistados sentiam desconforto, na região do quadril, durante o carregamento e descarregamento dos pallets no monta carga, atividade realizada uma vez por mês e $33 \%$, bem como, sentiam desconforto nos joelhos e no quadril durante o preparo da solução de $\mathrm{Ca}(\mathrm{OH})_{2}$, além das atividades o desconforto predominante era a dor, seguido de cansaço. Estes desconfortos foi apresentado por $67 \%$ dos entrevistados num período superior a 6 meses, sendo estes classificados de leve/ muito leve a moderado.

O desconforto para $67 \%$ dos colaboradores não aumentou durante a jornada de trabalho, mas aumentou para $33 \%$ dos entrevistados. Foi relatado que ninguém sente desconforto à noite ou durante as horas extras e este melhora durante o revezamento das tarefas, não necessitando de nenhum tipo de remédio para a maioria entrevistada.

\section{RULA X REBA}

No Quadro 3 são retratadas as situações de mudanças necessárias para cada tipo de atividade/ tarefa descrita de pertinência a este projeto, sendo contrapostas e simultaneamente complementadas as ferramentas RULA e REBA.

Quadro 3- Comparação dos resultados encontrados por meio das ferramentas REBA e RULA

\begin{tabular}{|l|l|l|}
\hline POSTURA/ ATIVIDADE & ANÁLISE REBA & ANÁLISE RULA \\
\hline Levantamento $1^{\circ}$ saco & $\begin{array}{l}\text { 10-Necessária o quanto } \\
\text { antes }\end{array}$ & $\begin{array}{l}\text { 7-Devem ser introduzidas } \\
\text { imediatamente }\end{array}$ \\
\hline Levantamento $2^{\circ}$ saco & $\begin{array}{l}\text { 10-Necessária o quanto } \\
\text { antes }\end{array}$ & $\begin{array}{l}\text { 7-Devem ser introduzidas } \\
\text { imediatamente }\end{array}$ \\
\hline Levantamento $3^{\circ}$ saco & $\begin{array}{l}\text { 10-Necessária o quanto } \\
\text { antes }\end{array}$ & $\begin{array}{l}\text { 7-Devem ser introduzidas } \\
\text { imediatamente }\end{array}$ \\
\hline Levantamento $4^{\circ}$ saco & 9-Necessária o quanto antes & $\begin{array}{l}\text { 7-Devem ser introduzidas } \\
\text { imediatamente }\end{array}$ \\
\hline Levantamento $5^{\circ}$ saco & 9-Necessária o quanto antes & $\begin{array}{l}\text { 7-Devem ser introduzidas } \\
\text { imediatamente }\end{array}$ \\
\hline Colocar no monta carga $1^{\circ}$ saco & 9-Necessária o quanto antes & $\begin{array}{l}\text { 7-Devem ser introduzidas } \\
\text { imediatamente }\end{array}$ \\
\hline Colocar no monta carga $4^{\circ}$ saco & 9-Necessária o quanto antes & $\begin{array}{l}\text { 7-Devem ser introduzidas } \\
\text { imediatamente }\end{array}$ \\
\hline Colocar no monta carga $5^{\circ}$ saco & 9-Necessária o quanto antes & $\begin{array}{l}\text { 7-Devem ser introduzidas } \\
\text { imediatamente }\end{array}$ \\
\hline
\end{tabular}




\begin{tabular}{|c|c|c|}
\hline Transporte manual da carga para o monta carga & 8-Necessária o quanto antes & $\begin{array}{l}\text { 7-Devem ser introduzidas } \\
\text { imediatamente }\end{array}$ \\
\hline Colocar no monta carga $2^{\circ}$ saco & 8-Necessária o quanto antes & $\begin{array}{l}\text { 7-Devem ser introduzidas } \\
\text { imediatamente }\end{array}$ \\
\hline Colocar no monta carga $3^{\circ}$ saco & 8-Necessária o quanto antes & $\begin{array}{l}\text { 7-Devem ser introduzidas } \\
\text { imediatamente }\end{array}$ \\
\hline Colocar no monta carga $7^{\circ}$ saco & 8-Necessária o quanto antes & 6-Devem ser introduzidas \\
\hline Retirar pallet do monta carga com a paleteira & 8-Necessária o quanto antes & O método não abrange \\
\hline Puxar a paleteira & 8-Necessária o quanto antes & O método não abrange \\
\hline Levantamento $8^{\circ}$ saco & 7-Necessária. & 5-Devem ser introduzidas. \\
\hline Levantamento $10^{\circ}$ saco & 7-Necessária. & 5-Devem ser introduzidas. \\
\hline Colocar no monta carga $6^{\circ}$ saco & 7-Necessária. & 6-Devem ser introduzidas. \\
\hline Levantamento $7^{\circ}$ saco & 6-Necessária. & 6-Devem ser introduzidas. \\
\hline Segurar o saco na tina de preparo de solução & 5-Necessária. & 6-Devem ser introduzidas. \\
\hline Corte do saco & 5-Necessária. & 4-Podem ser necessárias. \\
\hline Retirar $\mathrm{Ca}(\mathrm{OH})_{2}$ do saco & 5-Necessária. & 5-Devem ser introduzidas. \\
\hline Colocar o saco em local para posterior descarte & 5-Necessária. & 4-Podem ser necessárias. \\
\hline Cortar plástico involucro para fracionamento & 5-Necessária. & 3-Podem ser necessárias. \\
\hline Movimentar a paleteira até a área de estocagem & 5-Necessária. & 6-Devem ser introduzidas. \\
\hline Empurrar a paleteira & 5-Necessária. & 6-Devem ser introduzidas. \\
\hline Ajuste do saco na área de estocagem & 4-Necessária. & 6-Devem ser introduzidas. \\
\hline Movimentação do pallet para preparo de solução & 4-Necessária. & 6-Devem ser introduzidas. \\
\hline Juntar todos os sacos para descarte & 4-Necessária. & 3-Podem ser necessárias. \\
\hline Ajuste da paleteira & 3-Pode ser necessária. & 3-Podem ser necessárias. \\
\hline Pegar a mangueira & 2-Pode ser necessária. & 3-Podem ser necessárias. \\
\hline Jogar água na tina de preparo de solução & 2-Pode ser necessária. & 3-Podem ser necessárias. \\
\hline Lavar a área & 2-Pode ser necessária. & 2-Postura aceitável. \\
\hline
\end{tabular}

Fonte: COUTINHO e PINTO, 2014

\section{Estudo do Levantamento de Peso por NIOSH}

O objetivo ao utilizar o método NIOSH foi calcular as cargas máximas em condições desfavoráveis, a fim de prevenir e/ou mitigar a ocorrência de dores causadas durante atividades que solicitem o manuseio e transporte de cargas.

As análises aplicadas desde o primeiro até o oitavo saco de $\mathrm{Ca}(\mathrm{OH})_{2}$, posições que eram necessárias o levantamento e transporte de carga deram um IL superior a 1, sendo considerado ruim e com necessidade de mudanças.

\section{Partículas Respiratórias}

O resultado obtido por meio de teste realizado segundo a norma NHO 08: Coleta de Material Particulado Sólido Suspenso no Ar de Ambientes de Trabalho e NIOSH 0600 foi 26.863 mg/m³, quase 30 vezes maior, considerando o limite médio ponderado de $3 \mathrm{mg} / \mathrm{m}^{3}$. Como o particulado da cal hidratada é considerado respirável, os colaboradores envolvidos nesse processo estão expostos a doenças do grupo das pneumoconioses, já que existe a possibilidade do mesmo penetrar e se depositar além dos bronquíolos terminais (AVATEC, 2014). 


\section{Recomendações Ergonômicas}

Postura Adequada - Conscientizar os operadores de que a má postura durante um período longo será prejudicial a sua vida futura;

Fracionamento da carga - Verificar junto ao fornecedor da matéria prima a possibilidade de enviar a carga paletizada de forma a atender as necessidades da empresa.

Levantamento de carga - Recomenda-se que seja adaptado um regulador de altura que facilite no momento do preparo da solução de $\mathrm{Ca}(\mathrm{OH}) 2$. Existem no mercado paleteiras manuais e elétricas para elevação.

Elevador de carga - Construção de uma rampa no térreo para facilitar a entrada e saída de materiais do monta carga.

Emanação de particulado - Devido ao colaborador estar exposto a uma quantidade muito superior ao limite permitido, deve-se colocar um sistema coletor de pó, podendo este enviar o pó diretamente para a água bruta ou para o sistema de homogeneização da $\mathrm{Ca}(\mathrm{OH}) 2$.

Utilização do EPI - Recomenda-se uma máscara de classificação Peça Facial Filtrante 3 (PFF3), a fim de diminuir ainda mais a inalação do particulado.

\section{Considerações finais}

A análise ergonômica tem como finalidade identificar problemas na interação entre o homem, máquina e ambiente. As propostas para melhoria visam sempre o menor custo para a empresa, podendo ir desde a simples substituição de uma ferramenta de trabalho inadequada até a modificação do processo de seleção de um determinado segmento de atividade. É importante que as ações sejam ordenadas e conjuntas.

O presente Projeto foi iniciado na Empresa devido à necessidade de avaliar a exposição do colaborador ao particulado e, após a análise Ergonômica completa, chega-se ao entendimento de que existem outros pontos que também devem ser melhorados, alguns até com certa urgência.

Para o desenvolvimento da análise foram utilizados recursos tanto de base teórica, como NIOSH, REBA, RULA, quanto softwares, como Kinovea e Ergolândia. A partir destes, foi realizado um estudo em cima dos dados obtidos para a obtenção do diagnóstico.

Após o uso das ferramentas e softwares citados acima, chegou-se a conclusão de que o processo há muitas necessidades de alteração em prol do colaborador. Tendo como base este estudo, foram feitas recomendações ergonômicas como postura e uso de EPI adequado, fracionamento da carga, utilização de elevador de carga, entre outros; os quais atingem os objetivos de melhorar as condições do colaborador e da qualidade do processo. 
Foi constatada, por meio do projeto, a necessidade ainda existente em algumas empresas de conhecer mais sobre Ergonomia, visto que o estudo da área cognitiva e organizacional são tão importantes quanto às questões físicas e ambientais.

Portanto, por meio dessa sequência de estudos realizados, é visto a importância das empresas se conscientizarem que com estudos ergonômicos mais eficazes e eficientes em seus setores, além de prevenir a ocorrência de acidentes e afastamentos oriundos de doenças ocupacionais, a Ergonomia também as permite manterem-se competitivas no mercado, com um grupo de colaboradores mais satisfeitos e reduzindo até o tempo de processo.

\begin{abstract}
Reduce costs associated to a good work environment is the way to achieve a product with more quality and wellness at work, providing competitively and attractiveness to the final consumer. In the chemical industry, the reduction of ergonomic and environmental impacts during a process takes great importance to minimize damage to the company, employees, community and environment, leading to an economy and quality of life throughout the chain. Therefore the aim of the project was to evaluate the process of $\mathrm{Ca}(\mathrm{OH})_{2}$ extinction for raw water treatment

Among the main problems we have high exposure of the employee with the particulate emanating in the air, over-drive to perform an activity and water loss / product to clean the area after the process. The whole process was taken as a case study, for its development was applied Ergonomic Work Analysis, using ergonomic tools as RULA, REBA, NIOSH and NIOSH 0600.
\end{abstract}

Key-words: $\mathrm{Ca}(\mathrm{OH}) 2$, Ergonomic Analysis, Environment Analysis.

\title{
REFERÊNCIAS
}

ABERGO. O que é Ergonomia. [S.1,, 20--]. Disponível em: <http://www.abergo.org.br/internas.php? pg=o_que_e_ergonomia>. Acesso em: 22 maio 2014.

ABRAHÃO, J; SZNELWAR, L.I. SILVINO, A.; SARMET, M.; PINHO, D. Introdução à Ergonomia: da prática à teoria. São Paulo: Edgard Blucher, 2009. 240 p.

AGAHNEJAD, P. Análise ergonômica no posto de trabalho numa linha de produção utilizando método NIOSH: um estudo de caso no pólo industrial de Manaus. 2011. 92 p. Dissertação (Mestrado) - Universidade Federal do Pará, Instituto de Tecnologia, Belém, 2011.

AVATEC; Manual Prático: Insalubridade - Poeiras Minerais. 2014. Disponível em: <http://www.avatec.com.br/manual_insalubridade_poeira028.htm>. Acesso em: 06 set. 2014.

BARBOSA, F.; MATOS, L.; SANTOS, P. Determinação da concentração de partículas totais e respiráveis em suspensão no ambiente de trabalho. Ensaio de comparação entre laboratórios. Porto, Portugal, [19--]. Disponível em:

<https://www.google.com.br/url?sa=t\&rct=j\&q=\&esrc=s\&source=web\&cd=1\&cad=rja\&uact=8\&ved=0CCcQFjAA\&u $\mathrm{rl}=\mathrm{http} \% 3 \mathrm{~A} \% 2 \mathrm{~F} \% 2 \mathrm{Fwww}$. aramalhao.com\%2Fimg_upload\%2FEnsaio_ComparaCa(OH)2_entre_Laboratorios_Comuni $\mathrm{caCa}(\mathrm{OH}) 2 . p d f \& e i=c z W V U 6 X 6$ AqezsQS9koHABQ\&usg=AFQjCNEPZH5LizJyvlyRMhxzYyM8WCS8w\&sig2=Z3h hweff7wHI-Kpje0wlRQ\&bvm=bv.68445247,d.cWc>. Acesso em: 08 jun. 2014.

BATIZ; E. C.; VERGARA, L. G. L.; LICEA, O. E. A. Análise comparativa entre métodos de carregamento de cargas e análise postural de auxiliares de enfermagem, São Paulo, v. 22, n. 02. mar.-abr. 2012. Disponível em:

<http://www.scielo.br/scielo.php?pid=S0103-65132012000200008\&script=sci_arttext>Acesso em: 07 jun. 2014.

BAÚ, L. M. S.; Fisioterapia do Trabalho: Ergonomia, Legislação, Reabilitação. Curitiba: Clãdosilva, 2002. Disponível em: <http://www.excelenciaemgestao.org/portals/2/documents/cneg8/anais/t12_0496_3097.pdf> Acesso em: 05 jun. 2014. 
BUDA, J. F. Segurança e Higiene no Trabalho em Estações de Tratamento de Esgoto, São Paulo, v. 5, n. 2, p. 142-146. jul.- dez. 2004. Disponível em: <http://www2.ifsp.edu.br/edu/prp/sinergia/complemento/ sinergia_2004_n2/pdf_s/segmentos/artigo_08_v5_n2.pdf> Acesso em: 07 jun. 2014.

CDC CENTERS FOR DISEASE CONTROL AND PREVENTIONS. USA, 2014. Disponível em: <http://www.cdc.gov/niosh/>. Acesso em: 03 jun. 2014.

COSTA, L. G. Análise Ergonômica de Postos de Trabalho. [S.1.], 2014. Disponível em: <http://www.crpg.pt/empresas/recursos/kitergonomia/Documents/EWA_Portugu\%C3\%AAs_2004.pdf>. Acesso em: 30 maio 2014.

DIEGO-MÁS, J. A.; CUESTA, S. A. NIOSH (NATIONAL INSTITUTE for OCCUPATIONAL SAFETY and HEALTH). [S. 1.], 2014. Disponível em: 〈http://www.ergonautas.upv.es/metodos/niosh/niosh-ayuda.php>. Acesso em: 03 jun. 2014.

DOMINGUES, L. M.; SILVA, N. O. M.; SANTOS, R. E. Estudo Ergonômico para o Transporte de Cilindros de Hexafluoreto de Urânio. Resende: [s.n.], 2012. CD-ROM.

EHS CONSULTORIA E TREINAMENTO. São Paulo, 2014. Disponível em: <http://www.ehsconsultoria.com/consultoria.html>. Acesso em: 22 maio 2014.

FALZON, P. Ergonomia. São Paulo: Edgard Blucher, 2007. 640 p.

FUNDACENTRO. Ministério do Trabalho e Emprego. O Tamanho das Partículas de Poeira Suspensas no Ar dos Ambientes de Trabalho. [S. 1.], 2001. 97 p.

GUIMARÃES, B. P.; VERRI, N. S.; MARQUES,R. G. F. Análise Ergonômica de um Setor Administrativo de uma Indústria Nuclear. Resende: [s.n.], 2012. CD-ROM.

GUIMARÃES, C. P.; NAVEIRO, R. M. Revisão dos Métodos de análise ergonômica aplicados ao Estudo dos DORT em trabalho de montagem manual. Revista Produto e Produção, v. 7, n.01, p. 63-75, mar. 2004. Disponível em:

<http://www.seer.ufrgs.br/index.php/ProdutoProduCa(OH)2/ article/view/1431>. Acesso em: 26 maio 2014.

HIGNETT, S. McATAMNEY, L. Rapid Entire Body Assessment (REBA). Applied Ergonomics. USA: [s. n.], 2000. 31 : 201-205 p.

IIDA, I. Ergonomia, projeto e produção. São Paulo: Edgard Blucher, 2003. 465 p.

IPAC. Circular nº 8/2009. In C.O.D. LABORATÓRIOS. Instituto Português de Acreditação 2009.

LUEDER, R. A Proposed RULA for Computer Users. In: Proceedings of Ergonomics Summer Workshop. [S. 1], ago. 1996. Disponível em: <http://www.humanics-es.com/etc.htm>. Acesso em: 20 maio 2014.

MATIAS, N. T. Ergonomia. 2. Ed. Resende: Material Acadêmico, 2011. 49 p. Disponível em: <http://lore.com/Ergonomia.1> Acesso em: 02 jun. 2014.

NIOSH. NIOSH 05000 Total particulates not otherwise regulated. In NIOSH Manual of Analytical Methods, 1994.

NP1796:2007 Segurança e saúde do trabalho Valores limites de exposição profissional a agentes químicos.

OKIMOTO, M. L. L. R. Niosh. [S.1.], 2002. Disponível em: <https://www.google.com.br/\# $\mathrm{q}=$ Pegar+carga+pesada+com+o+tronco+em+flex\%C3\%A3o.+Ex.\%3A+pegar+sacos+com + material\%2C+quando+posi cionados+no+ch\%C3\%A3o>. Acesso em: 05 jun 2014.

\section{ORMELEZ, C. R; ULBRICHT, L. Análise Ergonômica do Trabalho Aplicada a um Posto de Trabalho com}

Sobrecarga Física. Revista Uniandrade, v. 11 , n. 02, p. 69-84, jul-dez. 2010. Disponível em:

$<$ http://www.google.com/url?sa=t\&rct=j\&q=\&esrc=s\&source=web\&cd=7\&cad=rja\&uact=882\&sqi=2\&ved=0CF4QFj AG\&url=http $\% 3 \mathrm{~A} \% 2 \mathrm{~F} \% 2 \mathrm{Fwww}$. uniandrade.br\%2Frevistauniandrade\%2Findex.php $\% 2$ Frevistauniandrade $\% 2 \mathrm{Farticle}$ \%2Fdownload\%2F13\%2F12\&ei=nlKFU-bLGqTMsQS1-YHgDQ\&usg=AFQjCNGcjWp5VPZWjYOvKs82b6ql1h-W4 Q\&sig2=EjIA1SG_4s0I8-5j18jK6g\&bvm=bv.67720277,d.cWc>. Acesso em: 30 maio 2014.

SILVA, H. D.; PEREIRA,T. R. D. S.; ANJOS, T. D. S.; SILVA, T. D.; MENESES,R.S. Ergonomia como Fator de Mudança na Produção do Trabalho Humano. In: CONGRESSO BRASILEIRO DE EDUCAÇÃO EM 
ENGENHARIA, 39, Blumenau, 2011. Disponível em:

<http://www.abenge.org.br/CobengeAnteriores/2011/sessoestec/art1842.pdf>. Acesso em 30 maio 2014.

STANTON, N.; HEDGE, A.; BROOKHUIS, K.; SALAS, E.; HENDRICK, H. Handbook of Human Factors and

Ergonomics Methods. Boca Raton, Londres, Nova Iorque, Washington DC: CRC Press 1960. Disponível em:

<http://lore.com/Orienta\%C3\%A7\%C3\%A3o.2>. Acesso em: 26 maio 2014.

VIEIRA, J. E. A. Gestão Ergonômica e Programas de Ergonomia na Empresa, 2014. Disponível em:

<http://www.ergonomia.ufpr.br/Ger\%20Ergon\%20JEV.pdf>. Acesso em: 30 maio 2014.

\section{Dados dos autores:}

\section{Nome completo: Camila Gávio Coutinho}

Filiação institucional: Universidade do Estado do Rio de Janeiro (UERJ)

Departamento: Departamento de Engenharia de Produção

Função ou cargo ocupado: Graduanda em Engenharia de Produção

Endereço completo para correspondência: UERJ, Rodovia Presidente Dutra km 298 - (sentido RJ -

SP). Polo Industrial - Resende - RJ.

Telefones para contato: (24) 993017052

e-mail: gavio.camila@gmail.com

\section{Nome completo: Francisco Santos Sabbadini}

Filiação institucional: Universidade do Estado do Rio de Janeiro (UERJ)

Departamento: Departamento de Engenharia de Produção

Função ou cargo ocupado: professor adjunto

Endereço completo para correspondência: UERJ, Rodovia Presidente Dutra km 298 - (sentido RJ SP). Polo Industrial - Resende - RJ.

Telefones para contato: (24) 33813889

e-mail: franciscosabbadini@gmail.com

\section{Nome completo: Mitaiana Maria de Carvalho Pinto}

Filiação institucional: Universidade do Estado do Rio de Janeiro (UERJ)

Departamento: Departamento de Engenharia de Produção,

Função ou cargo ocupado: Graduanda em Engenharia de Produção

Endereço completo para correspondência: UERJ, Rodovia Presidente Dutra km 298 - (sentido RJ SP). Polo Industrial - Resende - RJ.

Telefones para contato: (11) 944817880

e-mail:mitaiana@hotmail.com 
Nome completo: Nelson Tavares Matias

Filiação institucional: Universidade do Estado do Rio de Janeiro (UERJ)

Departamento: Departamento de Engenharia de Produção

Função ou cargo ocupado: Professor Adjunto

Endereço completo para correspondência: UERJ, Rodovia Presidente Dutra km 298 - (sentido RJ SP). Polo Industrial - Resende - RJ.

Telefones para contato: (24) 981162378

e-mail:nelson.matiaz@gmail.com

Nome completo: Rosinei Batista Ribeiro

Filiação institucional: Faculdade de Tecnologia do Estado de São Paulo, Unidade de Cruzeiro (IES)

Departamento: Departamento de Engenharia de Produção

Função ou cargo ocupado: Professor Associado

Endereço completo para correspondência: Rua Othon Barcellos s/n, Centro Cruzeiro/SP - 12730-010,

Brasil

Telefones para contato: (12) 21242850

e-mail: rosinei1971@gmail.com

Submissão: $31 / 09 / 2015$

Aceito: 06/03/2016 